\title{
Profesores universitarios de Contabilidad y el proceso de tutorización del trabajo fin de grado
}

\author{
José-Antonio Donoso-Anes, Francisco Serrano-Domínguez y José-Antonio Camúñez-Ruíz
}

\section{RESUMEN}

El trabajo recoge la opinión y percepción de los profesores universitarios de Contabilidad de España sobre los aspectos más relevantes relacionados con la materia de Trabajo Fin de Grado (TFG), en concreto sobre regulación, asunción de créditos, obligatoriedad para profesores y alumnos, dedicación y reconocimiento de créditos, asignación, tutorización, evaluación y competencias a desarrollar en el TFG. Además, se han estudiado los factores que influyen en el estado general de satisfacción. La metodología seguida ha sido detectar los temas que sobre TFG preocupan a los profesores, elaborar el cuestionario de prueba inicial y final y distribuirlo por internet. El análisis descriptivo y de correlaciones se realiza mediante Microsoft Excel y SPSS 23.0. El estudio de satisfacción se hace mediante análisis factorial exploratorio a través de SPSS 23.0. Los resultados muestran que existe un nivel general de no satisfacción, motivado por la no aceptación metodológica que conlleva el TFG y las propias autoexigencias del profesor cuando acomete la tutorización, ya que siente que los resultados obtenidos nunca están a la altura de sus demandas.

Palabras clave: profesores universitarios, profesor tutor, tesis de grado, satisfacción profesional, España.

José-Antonio Donoso-Anes

Español. Doctor en Ciencias Económicas y Empresariales, Universidad de Sevilla, España. Profesor Titular, Universidad de Sevilla. Temas de investigación: docencia de la contabilidad, entidades deportivas y sin ánimo de lucro, inteligencia emocional. ORCID: 0000-0001-7454-1078.

Francisco Serrano-Domínguez 


\title{
Professores universitários de Contabilidade e o processo de orientação do Trabajo Fin de Grado
}

\section{RESUMO}

O trabalho recolhe a opinião e percepção dos professores universitários de Contabilidade da Espanha sobre os aspectos mais relevantes relacionados com a matéria de Trabajo de Fin de Grado (TFG), em concreto sobre regulação, assunção de créditos, obrigatoriedade para professores e alunos, dedicação e reconhecimento de créditos, designação, orientação, avaliação e competências a desenvolver no TFG. Além disso, se estudou os fatores que influenciam no estado geral de satisfação. A metodologia seguida foi detectar os temas que sobre TFG preocupam aos professores, elaborar o questionário de prova inicial e final e distribui-los por internet. A análise descritiva e de correlações se realiza mediante Microsoft Excel e SPSS 23.0. O estudo de satisfação se faz mediante análise fatorial exploratório através de SPSS 23.0. Os resultados mostram que existe um nível geral de não satisfação, motivado pela não aceitação metodológica que acarreta o TFG e as próprias autoexigências do professor quando acomete a orientação, já que sente que os resultados obtidos nunca estão à altura de suas demandas.

Palavras chave: professores universitários, professor orientador, tese de graduação, satisfação profissional, Espanha.

\section{University teachers of Accounting and the tutoring process of the graduation project}

\begin{abstract}
This work gathers the opinions and perceptions of university teachers of the Accounting degree in Spain on the most relevant aspects related to the Final Degree Project (Trabajo de Fin de Grado, TFG), specifically on regulation, assumption of credits, obligatory nature for professors and students, dedication and recognition of credits, assignment, tutoring, evaluation and competencies to be developed in the TFG. In addition, the factors influencing the general state of satisfaction were studied. The chosen methodology was to detect the issues of concern for the professors about the TFG, to prepare the initial and final test questionnaire and to distribute it via the Internet. The descriptive and correlative analysis was carried out using Microsoft Excel and SPSS 23.0. The satisfaction study is carried out by means of exploratory factor analysis using SPSS 23.0. The results show that there is a general level of non-satisfaction, motivated by the methodological non-acceptance of the TFG and the professor's own self-demands when tutoring, since he/she feels that the results obtained never meet to his/her demands.
\end{abstract}

Keywords: university professors, professor tutor, undergraduate thesis, professional satisfaction, Spain.

Recepción: 25/04/18. Aprobación: 13/02/20. 


\section{Introducción}

Actualmente resulta necesario impulsar una formación que garantice que el estudiante salga al mercado laboral no sólo con dominio de conocimientos técnicos sino también de competencias demandadas por los empleadores (Ramírez y Medina, 2008). En la actualidad, dentro de los diseños curriculares, las prácticas en empresas y el trabajo fin de grado (TFG) ayudan a la obtención de dichas competencias (Malcolm, 2012; Rowley y Slack, 2004).

Un repaso a la literatura internacional sobre TFG nos permite agruparla en temáticas relacionadas con las competencias, la labor de tutela, la evaluación, el rendimiento, la relación enseñanza-aprendizaje. Se han realizado estudios sobre competencias que se esperan desarrollar a través de su realización (James, 1998), el desarrollo de la autonomía e independencia (Greenbank y Penketh, 2009; James, 1998), el manejo de los recursos bibliográficos y el uso correcto del lenguaje académico (Callison, 1997; Castelló et al., 2012; Jensen et al., 2004; Porter, 2011), potenciar las labores de investigación en aquellas titulaciones donde la investigación forma parte imprescindible de las competencias profesionales (Garde-Hansen y Calvert, 2007; James et al., 2011), el necesario enlace entre los conocimientos teóricos y su aplicación práctica (Rowley y Slack, 2004) o trabajar bajo presión y estrés (Devonport y Lane, 2006). Relacionado con la labor de tutela, citamos los trabajos que estudian las percepciones y expectativas de los tutores (Feather, Anchor y Cowton, 2014; Jamieson y Gray, 2006; Malcolm, 2012; Todd, Smith y Bannister, 2006; Torres et al., 2014; Woolhouse, 2002), los que analizan el papel desarrollado por el tutor (Armstrong y Shanker, 1983; Cook, 1980; Sanderson, Clewes y Hand, 1998), aquellos que se adentran en aspectos como el uso de la tecnología para la tutela (Arias, 2018; Augustsson y Jaldemark, 2014; Heinze y Heinze, 2009; Jaldemark y Lindberg, 2013; López y Flores, 2006) y los que acometen la búsqueda de sistemas de tutela efectivos (Rowley, 2000). Los que abordan el proceso de evaluación analizando los criterios generales (Bettany-Saltikov et al., 2009; Leonard, Fitzgerald y Riordan, 2016; Pathirage et al., 2007; Pathirage et al., 2004; Saunders y Davis, 1998; Todd et al., 2004; Webster et al., 2000) o criterios específicos para determinadas áreas de conocimiento como las finanzas y administración de empresas (Hand y Clewes, 2000) y cuando tienen más de una función académica (StarrGlass y Ali, 2012; Jiménez, Díaz y Catalá, 2011). Por último, otro conjunto de trabajos como los desarrollados por Holman y Banning (2012) que analizan las temáticas de los TFG con mejores calificaciones o el efecto en los procesos de enseñanza-aprendizaje de James (1998) y Rowe y Okell (2009).

En España, las publicaciones relacionadas con los TFG comienzan a partir de la aplicación del Real Decreto 1393/2007 que ordena las enseñanzas universitarias oficiales. Entre sus muchas novedades destaca la obligatoriedad de realizar un TFG como parte de un sistema de garantía de calidad dentro de la nueva era de la Educación Superior (Mateo et al., 2012). Destacan los trabajos de Briones y Vera (2013; 2015); Vera, Briones y Sotos (2014) y Vera y Briones (2015; 2016) enfocados en conocer la percepción de los estudiantes sobre los TFG en general y los sistemas de evaluación que se les aplican. Los trabajos relacionados con el proceso de evaluación de Romero et al., (2011) y de Bonilla et al. (2012); los centrados en el desarrollo y evaluación de competencias de Valderrama et al. (2010), Rullan et al. (2010), Rekalde (2011), Bonilla y Martín (2012), Félix et al., (2016) y Ruíz y Tirado (2016); así como los trabajos de Vilardell (2010), Delgado y Martín (2012), Bonilla et al. (2012), y Donoso et al., (2016) relacionados con el área de Ciencias Sociales y Jurídicas.

Un referente a tener en cuenta es el trabajo de Del Río et al., (2017) que, siguiendo la misma metodología del presente estudio pero aplicado a los estudiantes, acepta la hipótesis de que las competencias desarrolladas por el alumno en su interacción con su supervisor aumentan su satisfacción. En este caso, 
relaciona satisfacción del alumno con el desarrollo de competencias en la realización del TFG.

Este trabajo está motivado por la necesidad de conocer cómo están afrontando los profesores universitarios de Contabilidad el proceso de tutorización del TFG y su nivel de satisfacción. Este colectivo en España viene mostrando en diferentes foros su preocupación sobre aspectos concretos que afectan al desarrollo de los TFG. En consecuencia, el objetivo principal del trabajo es detectar qué factores son los que influyen en el grado de satisfacción de los profesores universitarios de Contabilidad en España a la hora de hacer frente al proceso de tutorización del TFG. Además de exponer las opiniones y percepciones de los profesores sobre el método de trabajo (el papel del tutor, orientación y contenido del trabajo, tutorización, aprobación de cierre y preparación de la defensa), la evaluación final del TFG y la importancia de las competencias a desarrollar por parte del alumno en el TFG y su nivel de desarrollo.

\section{Metodología}

En la primera fase se detectan los temas que preocupan a los profesores sobre TFG. En este sentido, se realizan un total de 15 reuniones: 12 individuales, con guión semiestructurado, con profesores universitarios de Contabilidad a nivel nacional; una en grupo de 40 profesores de las diferentes áreas de conocimiento, con guión abierto. Por último, dos reuniones en grupo de seis profesores de las áreas de conocimiento y comisiones de seguimiento de los TFG de la Facultad de Ciencias Económicas y Empresariales y de la Facultad de Turismo y Finanzas de la Universidad de Sevilla, con guión semiestructurado. Como consecuencia se obtuvo el listado de temas que preocupaban a los profesores, entre los que destacamos:

- Método de trabajo: papel del tutor, orientación y contenido del trabajo, tutorización, aprobación de cierre y preparación de la defensa.
- Evaluación final del TFG.

- Importancia de las competencias a desarrollar por parte de los alumnos en el TFG y su nivel de desarrollo.

Con el listado de problemas propuestos se elabora un cuestionario que recoge 74 entradas y se distribuye mediante Google Formulario. Se hacen 15 pruebas de control a profesores de Contabilidad, se corrige redacción y errores, y se elabora el cuestionario definitivo. ${ }^{1}$

\section{Muestra y características demográficas}

La población a la que va dirigido el cuestionario son los profesores universitarios de Contabilidad. Actualmente, según fuentes de la Asociación Española de Profesores Universitarios de Contabilidad (ASEPUC), su número a nivel nacional es de aproximadamente 900 . El cuestionario se ha distribuido a través de la base de datos de ASEPUC que recoge al 80\% del profesorado, de los directores de departamento y directamente a los profesores. El número de respuestas válidas ha sido de 247 de 48 universidades españolas (ver Anexo 1).

La relación entre el tamaño muestral y el tamaño poblacional viene dada, para el caso en el que se trata de estimar una proporción, por la igualdad (Lohr, 2000):

$$
n=\frac{N \cdot z_{\alpha}^{2} \cdot p \cdot q}{d^{2} \cdot(N-1)+z_{\alpha}^{2} \cdot p \cdot q}
$$

con $n=$ tamaño muestral, $\mathcal{N}=$ tamaño poblacional, con una confianza, $1-\boldsymbol{\alpha}$ que suponemos del $95 \%$, $1-\alpha=0,95$, para el cual se verifica $z_{\alpha}=1,96$ bajo el supuesto de normalidad. Con $p$, proporción que se quiere estimar. En el peor de los casos, $p=0,5$. Con $q=1-p$. A $p$ se le suele llamar heterogeneidad. Y con $d=$ margen de error, o también conocida como precisión, que en la igualdad anterior aparece en tanto por uno, pero a la hora de informar se expresa en tanto por ciento. Se acepta un margen de error entre el 1\% y el $9 \%$.

\footnotetext{
${ }^{1}$ Dirección del cuestionario https://forms.gle/qJxcRzSnZ7XhpfqD8
} 
En el caso que nos ocupa, podemos afirmar que con una población de tamaño 900 y una muestra de 247 individuos, para un nivel de confianza del 95\% y una heterogeneidad del $50 \%$ de los casos, se obtiene un margen de error de $5.31 \%$.

Con la información obtenida y mediante IBM SPSS Statistics 23.0, se ha realizado un análisis estadístico que ha comprendido estudio descriptivo, de correlaciones, de test de comparación de medias y análisis factorial para medir el estado de satisfacción.

La muestra recoge la opinión de las diferentes categorías profesionales (tabla 1) y una paridad de hombre-mujer (51\%, 126 hombres y el 49\%, 121 mujeres). El perfil del profesorado que ha contestado la encuesta es de profesores con experiencia. El 80\% tiene una antigüedad de más de 15 años, el 19\% ha dirigido tres o más tesis doctorales, el $46.2 \%$ ha dirigido tres o más trabajos fin de máster y el 88.7\% ha tenido la experiencia de tutorizar tres o más TFG. Se sienten capacitados y formados para hacer frente a la tutela del TFG, así lo perciben los propios profesores que valoran con una media de $4.4^{2}$ el sentirse capacitados y con $4.4^{3}$ el sentir que tienen formación suficiente para tutelar TFG.

Tabla 1. Categoría Profesional

\begin{tabular}{|l|c|c|}
\hline & $\boldsymbol{n}$ & \% \\
\hline Catedrático & 19 & 7.7 \\
\hline Titular Universidad & 100 & 40.5 \\
\hline Catedrático de Escuela Universitaria & 8 & 3.2 \\
\hline Titular de Escuela Universitaria & 33 & 13.4 \\
\hline Asociado & 12 & 4.9 \\
\hline Contratado Doctor & 33 & 13.4 \\
\hline Ayudante Doctor & 11 & 4.5 \\
\hline Colaborador & 21 & 8.5 \\
\hline Otros & 10 & 4.0 \\
\hline Total & $\mathbf{2 4 7}$ & $\mathbf{1 0 0 . 0}$ \\
\hline
\end{tabular}

Fuente: elaboración propia.

\section{Resultados}

El perfil tipo de dedicación del profesorado que tutoriza TFG es un docente que tutoriza cuatro TFG por curso académico. El número de reuniones por TFG es de dos a cinco y el tiempo medio de cada reunión está entre 30 y 45 minutos, por lo que el número de horas totales dedicadas a cada TFG, computando no sólo las horas de tutoría presencial sino también las revisiones, correcciones y preparación para la defensa, está entre 10 y 20 horas. A continuación, presentamos las opiniones y percepciones de los profesores sobre el método de trabajo, la evaluación y las competencias relacionadas con el TFG. Asímismo, identificamos los factores que influyen en el grado de satisfacción de los profesores universitarios de Contabilidad en España a la hora de hacer frente al proceso de tutorización del TFG.

${ }^{2}$ Apartado 57 del cuestionario. Media sobre una escala de Likert de 1 a 5 (1-muy en desacuerdo, 5-muy de acuerdo).

${ }^{3}$ Ídem. 


\section{Método de trabajo seguido por el profesor}

El análisis descriptivo de cómo afrontan los profesores el TFG abarca el papel del tutor, la orientación y contenido del trabajo, la tutorización, la aprobación de cierre y la preparación de la defensa.

La normativa expresa que el TFG lo realizará el estudiante bajo la dirección de un tutor y determina que la función del tutor consistirá en orientar al estudiante durante la realización del trabajo, supervisarlo y velar por el cumplimiento de los objetivos fijados.

Los profesores consideran que están realizando más un papel de dirección (53.4\%) que de mentorización, ya que se implican de forma más activa en todo el proceso de realización del trabajo y perciben que el resultado final es de su responsabilidad, por lo que su imagen y prestigio quedan expuestos públicamente. Este papel, en el ámbito académico universitario, es el que está asociado a trabajos con orientación de investigación como la dirección de tesis doctorales donde el alumno está más sujeto a las directrices que marca el profesor. Por otro lado, el 42.5\% de los profesores consideran que están desarrollando más una labor de mentorización del TFG, ya que su papel es más de orientador, guía o consejero y deja al alumno un alto grado de autonomía para que desarrolle las competencias de carácter instrumental y sistémicas asignadas a la asignatura. En este caso, el tutor hace que la responsabilidad del resultado final recaiga exclusivamente en el alumno, si bien el tutor aporta experiencia y conocimientos sobre el tema (Manzano et al., 2012). La opinión mayoritaria es que estos roles deberían invertirse; el 64.8\% entiende que se debería ser más mentor que director $(32.8 \%$ ) (tabla 2).

Respecto a la orientación que debe tener el contenido del TFG, la normativa lo deja abierto a diferentes modalidades, indica que podrá ser un proyecto, memoria o estudio sobre un tema, en el que el alumno desarrollará y aplicará conocimientos, capacidades y competencias adquiridas en la titulación.

Tabla 2. Papel del tutor

\begin{tabular}{|l|c|c|c|c|}
\hline & \multicolumn{2}{|c|}{ Lo que hago } & \multicolumn{2}{c|}{ Lo que debería hacer } \\
\hline Director del trabajo & $\mathbf{1 3 2}$ & $\mathbf{5 3 . 4 \%}$ & 81 & $32.8 \%$ \\
\hline Mentor del trabajo & 105 & $42.5 \%$ & $\mathbf{1 6 0}$ & $\mathbf{6 4 . 8 \%}$ \\
\hline Otro & 1 & $0.4 \%$ & 4 & $1.6 \%$ \\
\hline Total & $\mathbf{2 3 8}$ & $\mathbf{9 6 . 4 \%}$ & $\mathbf{2 4 5}$ & $\mathbf{9 9 . 2 \%}$ \\
\hline
\end{tabular}

Fuente: elaboración propia

La orientación que desea dar el tutor al TFG se correlaciona con la opinión sobre el papel que desea desempeñar. Así, a la pregunta ¿debe ser un trabajo que forme al alumno en la investigación, en su desarrollo profesional o una continuidad en su formación académica? Los resultados muestran que el 52.2\% de los profesores están orientando el TFG a un mixto de formación académica-profesional-investigación (tabla 3), pero los que desean apostar por este enfoque se incrementan hasta el 60.3\%. Los que están en el enfoque de preparación profesional son el 18.6\% y los que consideran deberían hacerlo aumentan al $25.9 \%$. Los incrementos anteriores se producen en detrimento de trabajos orientados a la continuidad de la formación académica que pasa del 19.8\% al $10.9 \%$ y de la formación investigadora que pasa del $6.5 \%$ al $1.2 \%$ ya que consideran que los alumnos durante el Grado no han adquirido competencias vinculadas a la investigación y, por lo tanto, correlaciona con la opinión de desarrollar más un papel 
de mentor (orientador) en detrimento del de director (guía de investigación) (Quevedo y Rodrigues, 2010). Por lo tanto, no se centran en este tipo de trabajos, aunque sí desarrollan aspectos parciales de metodología de investigación como búsquedas y revisiones bibliográficas.

Tabla 3. Orientación del TFG

\begin{tabular}{|l|c|c|c|c|}
\hline & \multicolumn{2}{|c|}{ Lo que hago } & \multicolumn{2}{c|}{ Lo que debería hacer } \\
\hline Preparación profesional & 46 & $18.6 \%$ & 64 & $25.9 \%$ \\
\hline Continuación de la formación académica & 49 & $19.8 \%$ & 27 & $10.9 \%$ \\
\hline Formación investigadora & 16 & $6.5 \%$ & 3 & $1.2 \%$ \\
\hline Mixto de las anteriores respuestas & $\mathbf{1 2 9}$ & $\mathbf{5 2 . 2 \%}$ & $\mathbf{1 4 9}$ & $\mathbf{6 0 . 3} \%$ \\
\hline Total & $\mathbf{2 4 0}$ & $\mathbf{9 7 . 2 \%}$ & $\mathbf{2 4 3}$ & $\mathbf{9 8 . 4 \%}$ \\
\hline
\end{tabular}

Fuente: elaboración propia.

En suma, al ser un trabajo finalista de formación académica, se busca romper con la continuidad del aprendizaje académico y hacer que a través del TFG el alumno se enfrente con la realidad profesional.

Respecto al contenido, el objetivo a alcanzar en la realización del TFG es desarrollar la integración de conocimientos adquiridos a lo largo de las diferentes materias que ha cursado. Por lo tanto, el TFG debería tener un enfoque multidisciplinar. Como apuntan Ferrer et al., (2013), el TFG multidisciplinar debe ser uno de los principales retos y dificultades, tanto para los alumnos, que generalmente están acostumbrados a trabajar con conocimientos parcelados, como para el profesor, que suele estar especializado en líneas de trabajo muy concretas.

En contenidos priman los trabajos monodisciplinares $(54.3 \%)$ sobre los multidisciplinares $(42.1 \%)$ aunque el $65.6 \%$ reconoce que deberían incrementarse estos últimos (tabla 4). La diferencia se justifica porque el profesor considera que los trabajos multidisciplinares requieren mayor dedicación y esto, unido al número elevado de TFG a tutorizar y su bajo reconocimiento en créditos, hace que el profesor se apoye más en temas monodisciplinares que son los que desarrollan contenidos que mejor domina y que le permitirán maximizar el tiempo de supervisión del trabajo.

Tabla 4. Contenido de los TFG

\begin{tabular}{|l|c|c|c|c|}
\hline & \multicolumn{2}{|c|}{ Lo que hago } & \multicolumn{2}{c|}{ Lo que debería hacer } \\
\hline Monodisciplinar & 134 & $\mathbf{5 4 . 3 \%}$ & 74 & $30.0 \%$ \\
\hline Multidisciplinar & 104 & $42.1 \%$ & $\mathbf{1 6 2}$ & $\mathbf{6 5 . 6 \%}$ \\
\hline Total & $\mathbf{2 3 8}$ & $\mathbf{9 6 . 4 \%}$ & $\mathbf{2 3 6}$ & $\mathbf{9 5 . 5 \%}$ \\
\hline
\end{tabular}

Fuente: elaboración propia. 
El hecho de que los trabajos estén relacionados con la especialización del tutor, hace que el alumno tenga un papel poco relevante en la elección del tema (tabla 5). De hecho, el alumno sólo elige en el 7.3\% y en el $35.2 \%$ lo hace el tutor. La modalidad más aceptada es mediante consenso tutor-alumno, en la que el tutor hace una propuesta de varios temas, pero siempre dentro de su singularidad y consensua la elección final; esta modalidad es la más utilizada $(54.3 \%)$ y la que el profesorado propone que se potencie $(70.4 \%)$.

El perfil actual de tutorización (tabla 6) es un profesor que atiende al alumno de forma individualizada, fija una planificación de seguimiento presencial y por internet, y facilita información metodológica y material bibliográfico.

Tabla 5. El tema del TFG lo propone

\begin{tabular}{|l|c|c|c|c|}
\hline & \multicolumn{2}{|c|}{ Lo que hago } & \multicolumn{2}{c|}{ Lo que debería hacer } \\
\hline El tutor & 87 & $35.2 \%$ & 46 & $18.6 \%$ \\
\hline El alumno & 18 & $7.3 \%$ & 23 & $9.3 \%$ \\
\hline Se ha de consensuar entre el tutor y el alumno & $\mathbf{1 3 4}$ & $\mathbf{5 4 . 3} \%$ & $\mathbf{1 7 4}$ & $\mathbf{7 0 . 4 \%}$ \\
\hline Total & $\mathbf{2 3 9}$ & $\mathbf{9 6 . 8} \%$ & $\mathbf{2 4 3}$ & $\mathbf{9 8 . 4 \%}$ \\
\hline
\end{tabular}

Fuente: elaboración propia.

Tabla 6. Perfil de tutorización

\begin{tabular}{|l|c|}
\hline & $\%$ \\
\hline Atiende de forma individualizada & $73.70 \%$ \\
\hline Tipo de contacto mixto presencial-internet & $87.90 \%$ \\
\hline Seguimiento siempre periódico, según planificación y tanto presencial como por internet & $52.60 \%$ \\
\hline Ofrece información y acceso a material bibliográfico & $82.60 \%$ \\
\hline
\end{tabular}

Fuente: elaboración propia.

El perfil deseado modificaría sensiblemente la situación actual, pues buscaría una tutoría mixta, individualizada y en grupo (49.4\%), potenciando el contacto y seguimiento por internet $(91.9 \%)$, aumentando la autonomía del alumno en el proceso de seguimiento, que decida cuando desea pasar por la tutoría (17\%), lo que implica incrementar su responsabilidad en la planificación y el autoaprendizaje (22.7\%) en línea del papel de mentorización que desea desempeñar el tutor.

El momento de dar por finalizado el trabajo y aprobar su cierre para su presentación es percibido por el profesor como un acto de responsabilidad que compromete su prestigio profesional (media $3.5 ;{ }^{4}$ con valores modales de $4,36 \%$ y $5,21.5 \%$ ).

Los profesores están dando su aprobación cuando el TFG reúne la mayoría de sus exigencias $(68.8 \%)$ o bien, aunque no reúne sus exigencias, cuando saben que no va a suspender (19\%), ya que el hecho de que el alumno suspenda no es indiferente para el profesor $(57.5 \%)$ pues siente que es un suspenso también para él. Su opinión es que los trabajos no están alcanzado su nivel de exigencia (media 3.1) $)^{5}$ y que los alumnos abusan del copia-pega (media 3.5) ${ }^{6}$ lo que está influyendo en la construcción de su bajo nivel de satisfacción.

\footnotetext{
${ }^{4}$ Media sobre una escala de Likert de 1 a 5 (1-muy en desacuerdo, 5-muy de acuerdo).

${ }^{5}$ Ídem.

${ }^{6} \dot{I} d e m$.
} 
En el caso de defensa pública del TFG (el 79.7\% de las respuestas hacen defensa pública con tribunal), ¿qué papel desempeña el tutor en su preparación? El $67.6 \%$ realiza siempre una supervisión del material de presentación y $34.4 \%$ además hace ensayos para la defensa.

Respecto a la asistencia del profesor al acto de defensa del TFG, la opinión del 45.7\% es que se debería asistir siempre que se pueda, mientras que el $37.2 \%$ no considera necesaria la asistencia al acto, ya que dedica suficiente tiempo a todo el proceso de tutorización.

En resumen, la metodología deseada por el tutor del TFG presenta el siguiente perfil: el tema a desarrollar debería ser elegido por consenso con el alumno pero debería ser multidisciplinar y con orientación profesional. Desea desempeñar un papel de mentor con seguimiento de forma individualizada y potenciando la vía internet. No da por cerrado el trabajo hasta que no reúne sus exigencias y se involucra en su defensa.

\section{Evaluación}

La normativa española no hace una mención expresa al tema, dejando abierta la posibilidad de utilizar procesos de evaluación alternativos a la exposición oral en sesión pública. El artículo 12.3 del RD. 1393/2007, referente a los títulos de grado, indica que estas enseñanzas concluirán con la elaboración y defensa de un TFG, pero no señalan cómo será la evaluación.

Las universidades internamente han normalizado el proceso de evaluación. Así, la mayoría le han dado carácter de defensa pública con tribunal constituido por tres titulares y tres suplentes con plena capacidad de evaluación y siendo los centros los que deciden la obligatoriedad o no del informe del tutor como elemento de referencia.

Efectivamente la evaluación mayoritariamente se está realizando mediante la defensa en sesión pública y con un tribunal $(79.5 \%)$ que evalúa la totalidad del trabajo (52.9\%), o bien un porcentaje de la evaluación la realiza el tutor y el resto, el tribunal (26.4\%). Esta última modalidad es la que prefieren los profesores $(45.7 \%)$ por encima de que sea el tribunal únicamente $(19.6 \%)$ o el tutor en defensa pública $(17.5 \%)$. En la modalidad de evaluación mixta tutortribunal, en algunos centros, el tribunal sólo se reúne a petición del alumno, una vez el tutor ha dado por superado el trabajo, para subir la nota.

De los 124 profesores que han manifestado que en sus centros "evalúa un tribunal mediante defensa pública oral”, 41 (33\%) están de acuerdo con ese procedimiento, mientras que $55(44 \%)$ preferirían que un porcentaje de la nota la ponga el tutor y el resto el tribunal evaluador. De los 62 que han informado que en su centro la nota es la combinación de ambos porcentajes, 42 (68\%) están de acuerdo con ese procedimiento.

En el caso de evaluación por un tribunal, el 74.9\% de los profesores opina que el informe del tutor debería ser obligatorio, aunque en la actualidad sólo el $61.1 \%$ lo tienen como tal. Sobre su contenido, el $51.8 \%$ expresa que debería incluir una valoración general sobre la actividad realizada por el alumno, su grado de cumplimiento y una puntuación numérica.

En respuesta abierta, la opinión de los profesores es que el proceso de evaluación mediante tribunales se ha traducido en una sobrecarga de dedicación no reconocida ya que obliga, además de a la tutorización, a la lectura y evaluación de un número no definido de TFG, aspecto que suma a su bajo nivel de satisfacción.

Respecto a la composición de los tribunales evaluadores, el $28.7 \%$ de los profesores opina que no deberían formar parte de los mismos aquellos profesores vinculados a materias marginales en el grado y se manifiestan a favor de que esté formado exclusivamente por profesores del área de conocimiento o departamento relacionado con el TFG o, en su caso, por profesores conocedores de la materia si el TFG es multidisciplinar (60.3\%). 


\section{Competencias a desarrollar en el TFG}

En la asignatura de TFG el alumno debe desarrollar, según se recoge en las memorias de verificación de los títulos, competencias genéricas y específicas. Dentro de estas últimas se consideran las competencias instrumentales que se refieren al uso de herramientas para el aprendizaje y la formación, y las competencias sistémicas que están relacionadas con la visión de conjunto y la capacidad de gestionar adecuadamente la totalidad de la actuación. El 80.2\% de los profesores perciben que tienen un buen dominio de dichas competencias (media 4.1) ten preparados para desarrollarlas en los alumnos.

Para medir las competencias se ha utilizado el Cuestionario de Efectividad del Uso de Metodologías de Participación Activa (CEMPA) de Carrasco Gallego et al., (2015), que valora (en una escala Likert de 1, muy poco, a 5, mucho) la percepción de la importancia de que se desarrolle la competencia y nivel realmente desarrollado.

Las competencias genéricas valoran el grado de utilidad de cursar la materia TFG para el alumno. El resultado muestra que el profesorado considera que el TFG es útil para los alumnos (todos los ítems presentan medianas y modas con valor 4), ya que los implica en su propio aprendizaje (4), ayuda a desarrollar habilidades propias de la titulación (3.8) así como a contrastar los conocimientos aprendidos en el aula con su aplicación en situaciones reales (3.7).

Los profesores han valorado la importancia de cada competencia específica para alcanzar los objetivos del TFG y paralelamente han valorado su percepción sobre el nivel que han alcanzado los alumnos mediante su realización (tabla 7).

Tabla 7. Competencias específicas: instrumentales

\begin{tabular}{|l|c|c|c|}
\hline & Importancia media & Nivel medio & Diferencia \\
\hline Competencias instrumentales & $\mathbf{4 . 1 4}$ & $\mathbf{3 . 4 8}$ & $\mathbf{- 0 . 6 7}$ \\
\hline Organización del tiempo & 4.19 & 3.46 & $\mathbf{- 0 . 7 2}$ \\
\hline Resolución de problemas & 4.02 & 3.30 & $\mathbf{- 0 . 7 2}$ \\
\hline Toma de decisiones & 4.00 & 3.29 & $\mathbf{- 0 . 7 6}$ \\
\hline Planificación & 4.28 & 3.52 & -0.37 \\
\hline Uso ordenadores & 3.92 & 3.55 & -0.62 \\
\hline Gestión de bases de datos & 4.14 & 3.52 & -0.69 \\
\hline Comunicación verbal & 4.16 & 3.47 & $\mathbf{0 . 7 5}$ \\
\hline Comunicación escrita & 4.43 & 3.69 & $\mathbf{- 0 . 7 5}$ \\
\hline
\end{tabular}

Fuente: elaboración propia.

\footnotetext{
${ }^{7}$ Media sobre una escala de Likert de 1 a 5 (1-muy en desacuerdo, 5-muy de acuerdo).
} 
Los profesores universitarios de Contabilidad opinan que las competencias instrumentales tienen una gran importancia para alcanzar los objetivos del TFG (4.14) pero perciben que el nivel medio de desarrollo por parte del alumnado es de 3.48; por lo que marcan una necesidad de intervención de -0.67 para que se anule esa diferencia. Excepto el uso de ordenadores todas las demás obtienen una media de 4 o más. Destacan la comunicación escrita (4.43) y la planificación (4.28) seguidas de la comunicación oral (4.16) y la organización del tiempo (4.19); mientras la percepción del nivel de desarrollo de las diferentes competencias ninguna alcanza la media de 4 , siendo las de menor nivel y desarrollo la resolución de problemas (3.30) y la toma de decisiones (3.29). La diferencia entre nivel e importancia (todas negativas) apunta a una necesidad de intervención y corrección, ya que los profesores perciben que los alumnos no están alcanzando el nivel de competencia suficiente; las principales son la planificación (diferencia de -0.76) y la comunicación escrita (-0.75), seguidas por organización del tiempo (-0.73), resolución de problemas y toma de decisiones $(-0.72)$ y la que menos el uso de ordenadores (-0.37).

Entre importancia y nivel de las competencias instrumentales existe correlación positiva (tabla 8), las dos variables se correlacionan en sentido directo, aunque sus medias difieran en cualquiera de los ítems estudiados (en todos los casos con $\mathrm{p}=0.000$ ).

Tabla 8. Correlación entre la importancia y nivel de las competencias instrumentales

\begin{tabular}{|c|c|c|}
\hline & Correlación & p-valor \\
\hline Importancia de organización tiempo y nivel alcanzado en organización tiempo & 0.433 & 0.000 \\
\hline Importancia de resolución de problemas y nivel alcanzado en resolución de problemas & 0.398 & 0.000 \\
\hline Importancia de toma de decisiones y nivel alcanzado en toma de decisiones & 0.367 & 0.000 \\
\hline Importancia de planificación y nivel alcanzado en planificación & 0.392 & 0.000 \\
\hline Importancia de uso de ordenadores y nivel alcanzado en uso de ordenadores & 0.702 & 0.000 \\
\hline Importancia de gestión bases de datos y nivel alcanzado en gestión de bases de datos & 0.540 & 0.000 \\
\hline Importancia de comunicación verbal y nivel alcanzado en comunicación verbal & 0.583 & 0.000 \\
\hline Importancia de comunicación escrita y nivel alcanzado en comunicación escrita & 0.353 & 0.000 \\
\hline
\end{tabular}

Fuente: elaboración propia.

En todos los casos también existen diferencias significativas en las medias entre importancia y el nivel alcanzado (tabla 9).

Las competencias sistémicas (tabla 10) se valoran de menor importancia (3.54) si las comparamos con las instrumentales (4.14), destacan la estimulación intelectual (3.82) y la creatividad (3.74). Perciben que el nivel alcanzado es menor en la delegación (2.5). La diferencia entre nivel e importancia, excepto la delegación con -0.3 , todas presentan valores próximos a -0.7 , similar a las presentadas por las competencias instrumentales. Especialmente manifiestan una necesidad de intervención y corrección más alta la gestión por objetivos y la gestión de proyectos con -0.71.

Existe correlación positiva (tabla 11) en cualquiera de los ítems estudiados (en todos los casos con $\mathrm{p}=0.000$ ) entre la importancia y el nivel de las competencias sistémicas aunque las medias de nivel siempre están por debajo de las medias de importancia. En todos los casos existen diferencias significativas en las medias entre la importancia y el nivel alcanzado (tabla 12). 
Tabla 9. Test de comparación de medias para muestras pareadas de importancia y nivel

\begin{tabular}{|c|c|c|c|c|}
\hline & $\begin{array}{l}\text { Diferencia } \\
\text { de medias }\end{array}$ & $\begin{array}{c}\text { Desviación } \\
\text { estándar }\end{array}$ & Estadístico t & p-valor \\
\hline Importancia de organización tiempo y nivel alcanzado en organización tiempo & 0.727 & 1.013 & 11.069 & 0.000 \\
\hline Importancia de resolución de problemas y nivel alcanzado en resolución de problemas & 0.725 & 1.035 & 10.856 & 0.000 \\
\hline Importancia de toma de decisiones y nivel alcanzado en toma de decisiones & 0.725 & 1.082 & 10.380 & 0.000 \\
\hline Importancia de planificación y nivel alcanzado en planificación & 0.762 & 1.032 & 11.409 & 0.000 \\
\hline Importancia de uso de ordenadores y nivel alcanzado en uso de ordenadores & 0.374 & 0.779 & 7.408 & 0.000 \\
\hline Importancia de gestión bases de datos y nivel alcanzado en gestión de bases de datos & 0.617 & 0.912 & 10.477 & 0.000 \\
\hline Importancia de comunicación verbal y nivel alcanzado en comunicación verbal & 0.682 & 0.874 & 12.063 & 0.000 \\
\hline Importancia de comunicación escrita y nivel alcanzado en comunicación escrita & 0.750 & 0.966 & 12.029 & 0.000 \\
\hline
\end{tabular}

Tabla 10. Competencias específicas: sistémicas

\begin{tabular}{|l|c|c|c|}
\hline & Importancia media & Nivel medio & Diferencia \\
\hline Competencias sistémicas & $\mathbf{3 . 5 4}$ & $\mathbf{2 . 9 3}$ & $\mathbf{- 0 . 6 1}$ \\
\hline Creatividad & $\mathbf{3 . 7 4}$ & 3.08 & -0.66 \\
\hline Gestión por objetivos & 3.69 & 2.97 & $\mathbf{- 0 . 7 1}$ \\
\hline Gestión de proyectos & 3.63 & 2.92 & $\mathbf{- 0 . 7 1}$ \\
\hline Delegación & 2.82 & $\mathbf{2 . 5 1}$ & -0.31 \\
\hline Estimulación intelectual & $\mathbf{3 . 8 2}$ & 3.16 & -0.66 \\
\hline
\end{tabular}

Fuente: elaboración propia.

Tabla 11. Correlación entre la importancia y nivel de las competencias sistémicas

\begin{tabular}{|l|c|c|}
\hline & Correlación & p-valor \\
\hline Importancia de creatividad y nivel alcanzado en creatividad & 0.567 & 0.000 \\
\hline Importancia de gestión por objetivos y nivel alcanzado en gestión por objetivos & 0.433 & 0.000 \\
\hline Importancia de gestión por proyectos y nivel alcanzado en gestión por proyectos & 0.556 & 0.000 \\
\hline Importancia de delegación y nivel alcanzado en delegación & 0.630 & 0.000 \\
\hline Importancia de estimulación intelectual y nivel alcanzado en estimulación intelectual & 0.566 & 0.000 \\
\hline
\end{tabular}


Tabla 12. Test de comparación de medias para muestras pareadas entre importancia y nivel

\begin{tabular}{|l|c|c|c|c|}
\hline & $\begin{array}{c}\text { Diferencia } \\
\text { de medias }\end{array}$ & $\begin{array}{c}\text { Desviación } \\
\text { estándar }\end{array}$ & Estadístico t & p-valor \\
\hline Importancia de creatividad y nivel alcanzado en creatividad & 0.653 & 0.888 & 11.283 & 0.000 \\
\hline Importancia de gestión por objetivos y nivel alcanzado en gestión por objetivos & 0.703 & 1.005 & 10.755 & 0.000 \\
\hline Importancia de gestión por proyectos y nivel alcanzado en gestión por proyectos & 0.701 & 0.910 & 11.778 & 0.000 \\
\hline Importancia de delegación y nivel alcanzado en delegación & 0.312 & 0.860 & 5.551 & 0.000 \\
\hline Importancia de estimulación intelectual y nivel alcanzado en estimulación intelectual & 0.651 & 0.928 & 10.758 & 0.000 \\
\hline
\end{tabular}

Fuente: elaboración propia.

\section{Satisfacción del profesorado ante el TFG}

Los profesores muestran una baja satisfacción a la hora de valorar su experiencia del proceso de tutorización (dan una valoración media de satisfacción general de 2.9). ${ }^{8}$ Para el estudio de la satisfacción hemos seleccionado los apartados 58-70 del cuestionario (ver tabla 13) que recogen opiniones y percepciones del profesorado sobre su idoneidad de formación y competencias para afrontar la labor de tutorización, su estado reactivo de la emoción que interpreta la condición con la que afrontan la tarea, la correspondencia entre exigencia al alumno y resultados obtenidos, compromiso de su prestigio profesional, además de la relación entre el esfuerzo y dedicación de la tutorización y el reconocimiento en créditos.

Con estos 13 ítems se ha realizado un análisis factorial exploratorio, para agrupación de ítems, que ha generado cinco factores (ilustración 1) con índice de fiabilidad Alfa de Cronbach entre 0.59 y 0.88 .

Estos cinco factores los hemos denominado: percepción de competencia, aceptación metodológica, percepción de autoexigencia, prestigio profesional y reconocimiento (tabla 13).

Tabla 13. Factores de medida de la satisfacción

\begin{tabular}{|c|c|c|c|}
\hline Constructos & Items & Medias ${ }^{9}$ & $\begin{array}{l}\text { Alfa de } \\
\text { Cronbach }\end{array}$ \\
\hline \multirow{3}{*}{ Percepción de competencia } & 58. Me siento capacitado para tutelar TFG & 4.4 & \multirow{3}{*}{0.881} \\
\hline & 59. Tengo formación suficiente para tutelar TFG & 4.4 & \\
\hline & 63. Domino las competencias exigidas al alumnado para realizar TFG & 4.1 & \\
\hline \multirow{5}{*}{$\begin{array}{l}\text { Aceptación de la } \\
\text { metodología que conlleva } \\
\text { la materia de TFG }\end{array}$} & 60. La labor de tutela me aburre & 2.6 & \multirow{5}{*}{0.729} \\
\hline & 61. Considero la tutela una obligación & 3.8 & \\
\hline & 62. Tutelar me agobia & 2.5 & \\
\hline & 69. Prefiero impartir docencia a tutelar TFG & 3.7 & \\
\hline & 70. Tutelar TFG distorsiona mi actividad académica & 2.9 & \\
\hline
\end{tabular}

\footnotetext{
${ }^{8}$ Media sobre una escala de Likert de 1 a 5 (1-muy poca, 5-mucha).

${ }^{9}$ Media sobre una escala de Likert de 1 a 5 (1-muy en desacuerdo, 5-muy de acuerdo)
} 


\begin{tabular}{|l|l|l|}
\hline \multirow{2}{*}{$\begin{array}{l}\text { Percepción de } \\
\text { autoexigencias propias del } \\
\text { profesor }\end{array}$} & 64. Siento que los resultados obtenidos nunca están a la altura de mi exigencia & 3.1 \\
\cline { 2 - 3 } & 65. Siempre estoy alerta ya que siento que hay mucho fraude por copia-pega en el TFG & 3.5 \\
\hline Prestigio profesional & 66. Cuando doy la aprobación a un TFG comprometo mi prestigio como profesional & 3.5 \\
\cline { 2 - 3 } & 67. Si suspenden un TFG tutelado por mí me están suspendiendo a mí & 0.583 \\
\hline Reconocimiento & 68. La labor de tutela no está suficientemente reconocida & 2.8 \\
\hline
\end{tabular}

Fuente: elaboración propia.

\section{Análisis factorial 1.}

\section{Primer factor: percepción de competencia}

Un análisis factorial exploratorio de sus tres ítems (tabla 14) por el método de extracción Componentes principales, definen un único factor que hemos denominado Percepción de competencia que explica el $81 \%$ de la varianza de los tres ítems. El índice de fiabilidad
Alfa de Cronbach (Cronbach, 1951) toma el valor de 0.881 .

Las correlaciones (tabla 15) medidas por el coeficiente de correlación de Pearson, son significativas para los tres componentes, siendo la variable "Tengo formación suficiente" la que presenta un mayor coeficiente, 0.891 .

Tabla 14. Componentes percepción de competencia

\begin{tabular}{|l|c|c|c|}
\hline \multirow{2}{*}{} & \multicolumn{3}{|c|}{ Autovalores iniciales } \\
\cline { 2 - 4 } & Total & \% de varianza & \% acumulado \\
\hline 58. Me siento capacitado para tutelar TFG & $\mathbf{2 . 4 4 2}$ & $\mathbf{8 1 . 3 9 2}$ & $\mathbf{8 1 . 3 9 2}$ \\
\hline 59. Tengo formación suficiente para tutelar TFG & 0.449 & 14.963 & 96.355 \\
\hline 63. Domino las competencias exigidas al alumnado para realizar TFG & 0.109 & 3.645 & 100.000 \\
\hline
\end{tabular}

Fuente: elaboración propia.

Tabla 15. Correlaciones ( $p$-valor)

\begin{tabular}{|c|c|c|c|}
\hline & Me siento capacitado & Tengo formación suficiente & Domino las competencias \\
\hline \multirow{2}{*}{ Me siento capacitado } & 1.000 & $0.891^{* *}$ & $0.630^{* *}$ \\
\hline & & 0.000 & 0.000 \\
\hline \multirow{2}{*}{ Tengo formación suficiente } & $0.891^{* *}$ & 1.000 & $0.631^{* *}$ \\
\hline & 0.000 & & 0.000 \\
\hline \multirow{2}{*}{ Domino las competencias } & $0.630^{* *}$ & $0.631^{* *}$ & 1.000 \\
\hline & & 0.000 & \\
\hline
\end{tabular}

Fuente: elaboración propia. 
El factor mide la percepción que tiene el profesor sobre su nivel de competencia (técnica y no técnica) para hacer frente a la tutorización de los TFG. El resultado de medias $>4$ (tabla 13) da un perfil de profesorado que siente que tiene un alto nivel de preparación. Los tres ítems están bien correlacionados entre sí, lo que nos lleva a pensar que son el reflejo de un constructo o variable latente, la que define la preparación del profesor. El profesor se siente preparado para desarrollar esta tarea.

\section{Análisis factorial 2.}

\section{Segundo factor: Aceptación metodológica}

El análisis factorial exploratorio de sus cinco ítems (tabla 16) definen un único factor que hemos denominado Aceptación metodológica, que explica el 49\% de la varianza de los cinco ítems. El índice de fiabilidad Alfa de Cronbach toma el valor de 0.729.
Las correlaciones (tabla 17) son significativas para los cinco componentes. Por lo tanto, el factor mide el grado de aceptación del profesorado ante el hecho de tener que enfrentarse al proceso metodológico que conlleva la tutorización de los TFG. Los ítems recogen afirmaciones negativas con respecto a los demás ítems de la encuesta y con respecto a la satisfacción del profesor con esta tarea. Entre sí, los cinco ítems presentan correlaciones positivas y pueden considerarse un reflejo de la Aceptación metodológica por parte del profesorado. El resultado de medias se mueve entre 2.5 y 3.8 (tabla 13), por lo que el profesorado considera la tutorización del TFG una obligación más dentro de la actividad académica pero muestra su preferencia hacia la docencia sobre la tutorización de los TFG.

Tabla 16. Componente aceptación metodológica

\begin{tabular}{|l|c|c|c|}
\hline \multirow{2}{*}{} & \multicolumn{2}{|c|}{ Autovalores iniciales } \\
\cline { 2 - 4 } & Total & \% de varianza & \% acumulado \\
\hline 60. La labor de tutela me aburre & $\mathbf{2 . 4 4 3}$ & $\mathbf{4 8 . 8 6 8}$ & $\mathbf{4 8 . 8 6 8}$ \\
\hline 61. Considero la tutela una obligación & 0.958 & 19.160 & 68.028 \\
\hline 62. Tutelar me agobia & 0.642 & 12.841 & 80.869 \\
\hline 69. Prefiero impartir docencia a tutelar TFG & 0.538 & 10.763 & 91.632 \\
\hline 70. Tutelar TFG distorsiona mi actividad académica & 0.418 & 8.368 & 100.000 \\
\hline
\end{tabular}

Fuente: elaboración propia. 
Tabla 17. Correlaciones ( $p$-valor)

\begin{tabular}{|c|c|c|c|c|c|}
\hline & Tutelar me aburre & $\begin{array}{l}\text { La tutela es una } \\
\text { obligación }\end{array}$ & Tutelar me agobia & $\begin{array}{l}\text { Prefiero impartir } \\
\text { docencia }\end{array}$ & $\begin{array}{l}\text { La tutela de } \\
\text { TFG distorsiona } \\
\text { mi actividad } \\
\text { académica }\end{array}$ \\
\hline \multirow{2}{*}{ Tutelar me aburre } & 1.000 & $0.351^{* *}$ & $0.513^{* *}$ & $0.405^{* *}$ & $0.272^{* *}$ \\
\hline & & 0.000 & 0.000 & 0.000 & 0.000 \\
\hline \multirow{2}{*}{ La tutela es una obligación } & $0.351^{* *}$ & 1.000 & $0.282^{* *}$ & $0.178^{* *}$ & $0.141^{*}$ \\
\hline & 0.000 & & 0.000 & 0.006 & 0.029 \\
\hline \multirow{2}{*}{ Tutelar me agobia } & $0.513^{* *}$ & $0.282^{* *}$ & 1.000 & $0.438^{* *}$ & $0.454^{* *}$ \\
\hline & 0.000 & 0.000 & & 0.000 & 0.000 \\
\hline \multirow{2}{*}{ Prefiero impartir docencia } & $0.405^{* *}$ & $0.178^{* *}$ & $0.438^{* *}$ & 1.000 & $0.484^{* *}$ \\
\hline & 0.000 & 0.006 & 0.000 & & 0.000 \\
\hline \multirow{2}{*}{$\begin{array}{l}\text { La tutela de TFG distorsiona } \\
\text { mi actividad académica }\end{array}$} & $0.272^{* *}$ & $0.141^{*}$ & $0.454^{* *}$ & $0.84^{* *}$ & 1.000 \\
\hline & & 0.029 & 0.000 & 0.000 & \\
\hline
\end{tabular}

Fuente: elaboración propia.

\section{Análisis factorial 3.}

\section{Tercer, cuarto y quinto factor}

Con los cinco ítems que restan, de los 13 ítems de percepciones, se lleva a cabo el análisis factorial exploratorio. Se busca explicar más del 75\% de la variabilidad total de los ítems implicados, lo que nos lleva a la selección de tres factores que explican un $78.49 \%$ de la varianza (tabla 18). El índice de fiabilidad Alfa de Cronbach toma el valor de 0.583.

Tras efectuar una rotación Varimax, la matriz de componentes rotados (tabla 19) muestra la existencia de tres factores. El primero va asociado a los ítems: 64 .
"Siento que los resultados obtenidos nunca están a la altura de mi exigencia" y 65. "Siempre estoy alerta ya que siento que hay mucho fraude por copia-pega en el TFG", que forman el tercer factor al que denominamos Percepción de autoexigencia. El segundo lleva asociado los ítems: 66. "Cuando doy la aprobación a un TFG comprometo mi prestigio como profesional" y 67. "Si suspenden un TFG tutelado por mí me están suspendiendo a mí", que constituye el cuarto factor, Prestigio profesional. Por último, el tercero va asociado el ítem 68. "La labor de tutela no está suficientemente reconocida", que crea el quinto factor denominado Reconocimiento. 
Tabla 18. Componentes factores 3,4 y 5

\begin{tabular}{|c|c|c|c|}
\hline & \multicolumn{3}{|c|}{ Autovalores iniciales } \\
\hline & Total & $\%$ de varianza & $\%$ acumulado \\
\hline 64. Siento que los resultados obtenidos nunca están a la altura de mi exigencia & 1.908 & 38.153 & 38.153 \\
\hline 65. Siempre estoy alerta ya que siento que hay mucho fraude por copia-pega en el TFG & 1.118 & 22.367 & 60.520 \\
\hline 66. Cuando doy la aprobación a un TFG comprometo mi prestigio como profesional & 0.898 & 17.967 & 78.487 \\
\hline 67. Si suspenden un TFG tutelado por mí me están suspendiendo a mí & 0.719 & 14.384 & 92.871 \\
\hline 68. La labor de tutela no está suficientemente reconocida & 0.356 & 7.129 & 100.000 \\
\hline
\end{tabular}

Tabla 19. Matriz de componente rotado

\begin{tabular}{|l|c|c|c|}
\hline \multirow{2}{*}{} & \multicolumn{3}{|c|}{ Componentes } \\
\cline { 2 - 4 } & Factor 3 & Factor 4 & Factor 5 \\
\hline No se alcanza mi exigencia & -0.012 & $\mathbf{0 . 8 6 2}$ & -0.014 \\
\hline Estoy alerta con el copia-pega & 0.224 & $\mathbf{0 . 6 9 8}$ & 0.152 \\
\hline Comprometo mi prestigio & $\mathbf{0 . 8 8 3}$ & 0.162 & 0.058 \\
\hline Si suspende es como si suspendiera yo & $\mathbf{0 . 9 0 5}$ & 0.048 & 0.021 \\
\hline La tutela no está bien reconocida & 0.048 & 0.094 & $\mathbf{0 . 9 8 9}$ \\
\hline
\end{tabular}

Fuente: elaboración propia

La matriz de correlaciones (tabla 20) muestra las correlaciones significativas para los tres constructos. El factor de Percepción de autoexigencia presenta medias (tabla 13) de 3.1 y 3.5; por lo que los profesores muestran un moderado nivel de exigencia personal. El factor de Prestigio profesional (3.5) igualmente presenta un grado de preocupación del profesorado, no así con el sentimiento de reciprocidad en el caso de suspenso (2.8). Por último, el factor de Reconocimiento, con una media de 4.5 , evidencia el grado de enfrentamiento entre la dedicación en horas que asumen los profesores y el reconocimiento en créditos que reciben.

Se les han asignado puntuaciones a los cinco factores usando técnicas de regresión y a partir de los ítems que los definen, ello permite analizar las posibles relaciones de causa-efecto y medir las correlaciones entre esos factores y la variable Satisfacción (tabla 21), que resume en un dígito su opinión global sobre su tutorización de TFG y recordamos que la valoración media de satisfacción general es de 2.9 . 
Tabla 20. Correlaciones ( $p$-valor)

\begin{tabular}{|c|c|c|c|c|c|c|}
\hline & & $\begin{array}{c}\text { No se alcanza mi } \\
\text { exigencia }\end{array}$ & $\begin{array}{c}\text { Estoy alerta con el } \\
\text { copia-pega }\end{array}$ & $\begin{array}{c}\text { Comprometo mi } \\
\text { prestigio }\end{array}$ & $\begin{array}{c}\text { Si suspende } \\
\text { es como si } \\
\text { suspendiera yo }\end{array}$ & $\begin{array}{l}\text { La tutela no está } \\
\text { bien reconocida }\end{array}$ \\
\hline $\begin{array}{l}\text { No se alcanza mi } \\
\text { exigencia }\end{array}$ & \multirow{2}{*}{ F.3 } & 1 & $\begin{array}{c}0.263 \\
(0.000)\end{array}$ & $\begin{array}{c}0.154 \\
(0.008)\end{array}$ & $\begin{array}{c}0.090 \\
(0.080)\end{array}$ & $\begin{array}{c}0.118 \\
(0.032)\end{array}$ \\
\hline $\begin{array}{l}\text { Estoy alerta con el } \\
\text { copia-pega }\end{array}$ & & $\begin{array}{c}0.263 \\
(0.000)\end{array}$ & 1 & $\begin{array}{c}0.256 \\
(0.000)\end{array}$ & $\begin{array}{c}0.192 \\
(0.001)\end{array}$ & $\begin{array}{c}0.159 \\
(0.006)\end{array}$ \\
\hline $\begin{array}{l}\text { Comprometo mi } \\
\text { prestigio }\end{array}$ & \multirow{2}{*}{ F.4 } & $\begin{array}{c}0.154 \\
(0.008)\end{array}$ & $\begin{array}{c}0.256 \\
(0.000)\end{array}$ & 1 & $\begin{array}{c}0.638 \\
(0.000)\end{array}$ & $\begin{array}{c}0.118 \\
(0.032)\end{array}$ \\
\hline $\begin{array}{l}\text { Si suspende es como } \\
\text { si suspendiera yo }\end{array}$ & & $\begin{array}{c}0.090 \\
(0.080)\end{array}$ & $\begin{array}{c}0.192 \\
(0.001)\end{array}$ & $\begin{array}{c}0.638 \\
(0.000)\end{array}$ & 1 & $\begin{array}{c}0.083 \\
(0.096)\end{array}$ \\
\hline $\begin{array}{l}\text { La tutela no está bien } \\
\text { reconocida }\end{array}$ & F.5 & $\begin{array}{c}0.118 \\
(0.032)\end{array}$ & $\begin{array}{c}0.159 \\
(0.006)\end{array}$ & $\begin{array}{c}0.118 \\
(0.032)\end{array}$ & $\begin{array}{c}0.083 \\
(0.096)\end{array}$ & 1 \\
\hline
\end{tabular}

Fuente: elaboración propia.

Tabla 21. Correlaciones ( $p$-valor) entre factores y satisfacción general

\begin{tabular}{|l|c|}
\hline Percepción sobre competencias & $0.076 \quad(0.236)$ \\
\hline Aceptación metodológica & $-0.475^{* *}(0.000)$ \\
\hline Autoexigencia & $-0.223^{* *}(0.000)$ \\
\hline Prestigio profesional & $0.108 \quad(0.091)$ \\
\hline Reconocimiento & $-0.104 \quad(0.103)$ \\
\hline ** La correlación es significativa en el nivel 0.01 (2 colas). \\
\hline Fuente: elaboración propia.
\end{tabular}

Los resultados muestran que existe correlación significativa de los factores 2 y 3, Aceptación metodológica y Autoexigencia, con respecto a la opinión final de Satisfacción; por lo tanto, para el profesor estos son los factores de su estado reactivo emocional que interpreta la condición con la que afrontan la tarea y la falta de correspondencia entre lo que se le está exigiendo al alumno y el resultado final obtenido en el TFG.

La ilustración 1 recoge un esquema de posibles relaciones entre los constructos (considerados como variables explicativas) y la Satisfacción general del profesorado. En el mismo, se admite la existencia de correlaciones entre los constructos, que aparecen estimadas sobre las líneas de doble flecha. En las líneas de una flecha se recogen las relaciones causa-efecto y sus pendientes estimadas. El esquema muestra que los ítems son el reflejo de los constructos o variables latentes (son constructos "reflectivos", como se le denomina en la literatura). Las pendientes de los constructos sobre la Satisfacción general miden su grado de influencia sobre la misma (significativa o no), así como su signo. 
Ilustración 1. Representación del análisis factorial exploratorio

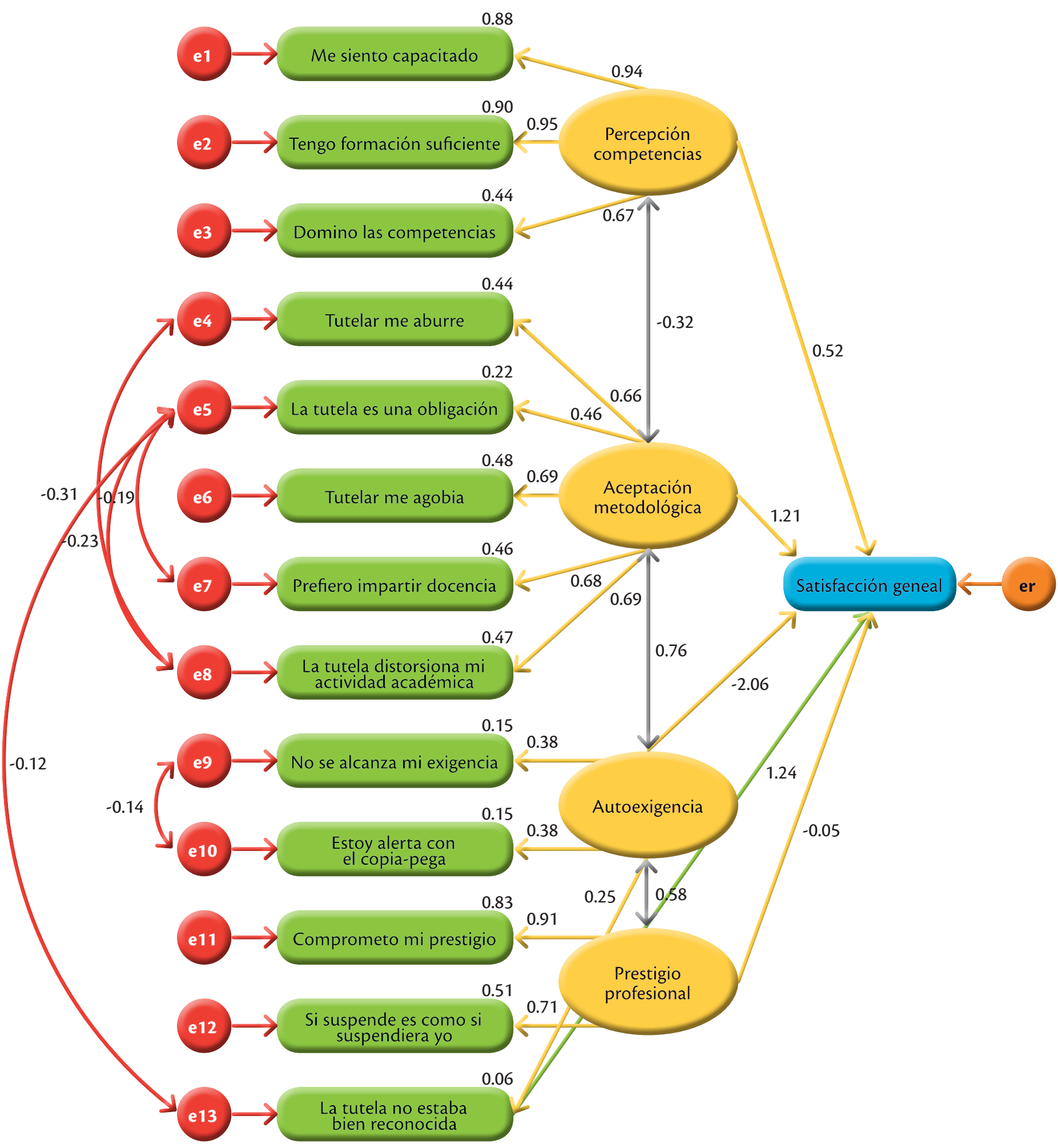




\section{Conclusión}

El trabajo recoge el estado opinión, percepción y satisfacción de los profesores universitarios de Contabilidad en España sobre aspectos relevantes relacionados con el TFG, en concreto tutorización, evaluación y competencias a desarrollar en el TFG.

Los profesores universitarios de Contabilidad no están satisfechos con su experiencia en la materia de TFG (valoración de satisfacción general 2.9). Tras la realización del análisis factorial exploratorio los constructos que la explican son la alta autoexigencia y la no aceptación metodológica. Respecto a la alta autoexigencia, el profesorado siente que los resultados no alcanzan sus exigencias que están centradas en las expectativas esperadas respecto a las competencias genéricas, instrumentales y sistémicas, así como el nivel desarrollado por los alumnos.

$\mathrm{El}$ profesor tiene expectativas muy altas respecto a lo que va a significar el TFG para el alumno. Considera que el TFG es muy útil para el alumno porque le implica en su propio aprendizaje, le ayuda a desarrollar habilidades propias de la titulación y le pone en contacto con situaciones reales. Por lo tanto, espera que el alumno desarrolle un alto nivel de las competencias instrumentales y sistémicas; pero la realidad percibida a la que se enfrenta es muy diferente, ya que observa cómo el alumno a través de la realización del TFG no está alcanzando el nivel exigido, por lo que correlaciona negativamente con el factor de autoexigencia y en consecuencia con su nivel de satisfacción. En competencias instrumentales marca un diferencial significativo en todos los ítems excepto en el uso de ordenadores y en especial en planificación, comunicación escrita, organización del tiempo, resolución de problemas y toma de decisiones. En competencias sistémicas las expectativas no se cumplen en gestión por objetivos y de proyectos.

La no aceptación metodológica lleva al profesor a alejarse de la tutela de TFG prefiriendo impartir clases, pues dedicarse a tutorizar TFG lo considera una distorsión a su tarea académica, asumiendo un papel emocional bajo que le lleva al aburrimiento y el agobio y, en consecuencia, a una baja satisfacción.

Los motivos que llevan al profesor a la no aceptación metodológica los encontramos en las diferencias entre lo que hace y desea hacer respecto al papel que desempeña, la orientación del trabajo y su evaluación. El profesor prefiere desempeñar un papel de mentorización, en vez de dirección, que el TFG tenga una orientación mixta académica-profesionalinvestigación y con preferencia a un contenido multidisciplinar, mientras que la orientación actual es más académica y monodisciplinar. Respecto al procedimiento de evaluación, siente la presión de que el resultado del trabajo lo está evaluando a él también, exponiendo públicamente su imagen y prestigio.

Todo lo apuntado, unido a que el profesor no siente que exista un adecuado reconocimiento a su esfuerzo y dedicación, genera la baja satisfacción.

La implicación de esta conclusión es que si queremos mejorar la satisfacción del profesorado respecto a la tutorización del TFG debemos hacer frente a varios aspectos:

- Construir una estrategia docente que pase por potenciar el papel de mentorización, con la inclusión de temas multidisciplinares que potencien todo el conjunto de competencias instrumentales y sistémicas.

- Que la evaluación final del trabajo se haga teniendo en cuenta la opinión y valoración del tutor.

- Que se incremente el reconocimiento en créditos a la dedicación.

\section{Contribución e implicaciones}

A lo apuntado en el apartado de conclusiones, podemos añadir que el trabajo contribuye a reducir la brecha entre la investigación en docencia de la Contabilidad y la práctica docente. Estos trabajos son bien recibidos por la literatura en investigación en docencia de la Contabilidad ya que palían la preocupación 
manifestada por autores como Apostolou et al. (2019); Jackling et al. (2013); Ravenscroft et al. (2008) y Rebele y St. Pierre (2015). Además, el trabajo contribuye a abrir la línea de investigación sobre modelización de la satisfacción del profesorado universitario en el contexto del uso de metodologías de participación activa, en concreto, para poder generar satisfacción en metodologías como aprendizaje basado en proyectos, casos y trabajos. Asímismo, ayuda al éxito de la relación profesor-alumno en los procesos que impliquen ayuda, asesoramiento y mentorización; además, el modelo tiene el potencial de poder ser utilizado en estudios de nuevas investigaciones a mayor escala y en otras áreas de conocimiento.

Igualmente, el trabajo tiene muchas posibilidades para ser utilizado en mediciones de satisfacción en la realización del TFG por parte de los alumnos y

\section{Referencias}

Apostolou, B., J. W. Dorminey, J. M. Hassell y A. Hickey (2019), "Accounting education literature review (2018)", Fournal of Accounting Education, 47, pp. 1-27, DOI: https://doi.org/10.1016/j.jaccedu.2019.02.001.

Arias, F. G. (2018), "Metodología para la valoración de resultados en trabajos finales de grado (TFG) de áreas tecnológicas", REDU. Revista de Docencia Universitaria, 16(2), p.177, DOI: https://doi.org/10.4995/ redu.2018.10189.

Armstrong, M. y V. Shanker (1983), "The supervision of undergraduate research: student perceptions of the supervisor role", Studies in Higher Education, 8(2), pp. 177-183, DOI: https://doi.org/10.1080/0307507831 2331379044 .

Augustsson, G. y J. Jaldemark (2014), “Online supervision: a theory of supervisors' strategic communicative influence on student dissertations", Higher Education, 67(1), pp. 19-33, DOI: https://doi.org/10.1007/ s 10734-013-9638-4. servir de contraste a trabajos ya realizados en esta línea como el estudio de Del Río, Díaz-Vázquez y Maside (2017).

En la misma línea, el modelo es compatible para estudios que comparen la misma variable satisfacción tanto para el profesor como para el alumno en la búsqueda de los elementos de coincidencia y diferencia (Jamieson y Gray, 2006).

\section{Limitaciones}

Además de las limitaciones inherentes a un estudio basado en cuestionarios autoadministrados y el uso de la escala Likert, están las relacionadas con las características de la muestra, que sólo utiliza profesores universitarios de Contabilidad de un solo país, lo que puede limitar la generalización de los hallazgos más allá de esta muestra.

Bettany-Saltikov, J., S. Kilinc y K. Stow (2009), "Bones, boys, bombs and booze: an exploratory study of the reliability of marking dissertations across disciplines", Assessment \& Evaluation in Higher Education, 34(6), pp. 621-639, DOI: https://doi.org/10.1080/02602930802302196.

Bonilla-Delgado,I.M.yC.Martín-López(2012), “Evaluación de competencias en el Trabajo Fin de Grado en Administración y Dirección de Empresas: una propuesta de la Facultad de Ciencias Sociales de Talavera de la Reina UCLM", Revista de Formación e Innovación Educativa Universitaria, 5(4), pp. 241-253.

Bonilla, M. J., L. Fuentes, C. Vacas y T. Vacas (2012), “An analysis of the Final Dissertation Assessment Process in new degrees", Educade: Revista de Educación En Contabilidad, Finanzas Y Administración de Empresas, (3), pp. 5-21.

Briones, E. y J. Vera (2013), "Sistemas de evaluación del TFG y satisfacción de los estudiantes de distintas áreas de conocimiento", en XII Foro sobre Evaluación de la Calidad de la Investigación y de la Educación Superior. Sevilla, España. 
Briones, E. y J. Vera (2015), "Sistemas de evaluación del Trabajo de Fin de Grado y satisfacción de los estudiantes de distintas áreas de conocimiento", en XII Foro Internacional sobre la Evaculación de la Calidiad de la Investigación y de la Educación Superior (FECIES), Sevilla, España.

Callison, D. (1997), "Evolution of methods to measure student information use", Library E Information Science Research, 19(4), pp. 347-357.

Carrasco, A., Donoso, J. A. Duarte, J. J. Hernández y R. López (2015), "Diseño y validación de un cuestionario que mide la percepción de efectividad del uso de metodologías de participación activa (CEMPA). El caso del Aprendizaje Basado en Proyectos (ABPrj) en la docencia de la contabilidad", Innovar, 25(58), pp. 143-158.

Castelló, M., A. Iñesta, M. Pardo, E. Liesa y R. Martínez-Fernández (2012), "Tutoring the end-ofstudies dissertation: Helping psychology students find their academic voice when revising academic texts", Higher Education, 63(1), pp. 97-115, DOI: https://doi. org/10.1007/s10734-011-9428-9.

Cook, M. G. F. (1980), "The role of the academic supervisor for undergraduate dissertations in science and science-related subjects", Studies in Higher Education, 5(2), pp. 173-185, DOI: https://doi.org/10.1080/0307 5078012331377206.

Cronbach, L. J. (1951), "Coefficient alpha and the internal structure of tests", Psychometrika, 16(3), pp. 297-334, DOI: https://doi.org/10.1007/BF02310555>.

Del Río, M. L., R. Díaz-Vázquez y J. M. Maside (2017), "Satisfaction with the supervision of undergraduate dissertations", Active Learning in Higher Education, 1(14), pp. 1-14, DOI: https://doi. org/10.1177/1469787417721365.

Delgado, I. y C. Martín (2012), “Evaluación de competencias en el Trabajo Fin de Grado en Administración y Dirección de Empresas: una propuesta de la Facultad de Ciencias Sociales de Talavera de la Reina UCLM" ("Evaluation of competence in the Final Project in Bussiness Administrati"), Revista de Formación E Innovación Educativa Universitaria, 5(4), pp. 241-253.
Devonport, T. J. y A. M. Lane (2006), "Cognitive appraisal of dissertation stress among undergraduate students", Psychological Record, 56(2), pp. 259-266, <http://www.scopus.com/inward/record.url?eid=2s2.0-33744740692\&partnerID=tZOtx3yl>.

Donoso-Anes, J. A., F. Serrano-Domínguez y J. A. Camúñez-Ruiz (2016), "Trabajo de Fin de Grado a debate entre los profesores del Área de Ciencias Sociales y Jurídicas de la Universidad de Sevilla", Educade Revista, 7, pp. 41-58.

Feather, D., J. R. Anchor y C. J. Cowton (2014), "Supervisors' perceptions of the value of the undergraduate dissertation", International fournal of Management Education, pp. 14-21, DOI: https://doi. org/10.1016/j.ijme.2013.06.002.

Félix, M., A. Romero y A. Guerrero (2016), “Competencias en Trabajos Fin de Grado: desarrollo y evaluación", Revista Iberoamericana de Educación, 71(1), pp. 29-44.

Ferrer, V., M. Carmona y V. Soria (2013), El trabajo de fin de grado: guía para estudiantes, docentes y agentes colaboradores, McGraw-Hill España, <https://dialnet.unirioja.es/ servlet/libro?codigo $=504065>$.

Garde-Hansen, J. y B. Calvert (2007), "Developing a research culture in the undergraduate curriculum", Active Learning in Higher Education, 8(2), pp. 105-116, DOI: https://doi.org/10.1177/1469787407077984.

Greenbank, P. y C. Penketh (2009), "Student autonomy and reflections on researching and writing the undergraduate dissertation", fournal of Further and Higher Education, vol. 33, núm. 4, pp. 463-472, DOI: https://doi.org/10.1080/03098770903272537.

Hand, L. y D. Clewes (2000), "Marking the difference: an investigation of the criteria used for assessing undergraduate dissertations in a business school", Assessment and Evaluation in Higher Education, 25(1), pp. 5-21, DOI: https://doi.org/10.1080/713611416.

Heinze, A. y B. Heinze (2009), "Blended e-learning skeleton of conversation: improving formative assessment in undergraduate dissertation supervision", British Journal of Educational Technology, 40(2), pp. 294-305, DOI: https://doi.org/10.1111/j.1467-8535.2008.00923.x>. 
Holman, D. K. y J. Banning (2012), "Honors Dissertation Abstracts: a bounded qualitative meta-study Honors Dissertation Abstracts", Fournal of the National Collegiate Honors Council, p. 338, <https://files.eric.ed.gov/ fulltext/EJ1082334.pdf>.

Jackling, B., R. Natoli, S. Nuryanah y D. Ekanayake (2013), "Celebrating 20 Years of Publication of Accounting Education: an international journal: 19922011 ", Accounting Education, 22(1), pp. 18-43, DOI: https://doi.org/10.1080/09639284.2012.755008.

Jaldemark, J. y J. O. Lindberg (2013), “Technologymediated supervision of undergraduate students' dissertations", Studies in Higher Education, 38(9), 13821392, DOI: https://doi.org/10.1080/03075079.2011 .626851 .

James, E., C. Vinten, E. Wood y D. Merrick (2011), "University undergraduate projects can enhance sixthform science teaching", School Science Review, 93(343), pp. 105-107.

James, P. (1998), "Progressive development of deep learning skills through undergraduate and postgraduate dissertations", Educational Studies, 24(1), pp. 95-105, DOI: https://doi.org/10.1080/0305569980240107.

Jamieson, S. y C. Gray (2006), "The supervision of undergraduate research students: expectations of student and supervisor", Practice and Evidence of Scholarship of Teaching and Learning in Higher Education, 1(1), pp. 37-59.

Jensen, B. E., K. A. Martin, B. L. Mann y T. Fogarty (2004), "Easing your pain: a method for evaluating research writing from students", en Measurement in Physical Education and Exercise Science, vol. 8, núm. 1, pp. 43-52, DOI: https://doi.org/10.1207/s15327841mpee0801_4.

Jiménez-Padilla, T., B. Díaz-Rodríguez y O. S. CataláConcepción (2011), "La preparación para la culminación de estudios de Contabilidad y Finanzas de la SUM, Cabaiguán, Cuba", Revista Iberoamericana de Educación Superior, vol. 2, núm. 5, pp. 126-140, DOI: https://doi.org/10.22201/iisue.20072872e.2011.5.

Leonard, S. N., R. N. Fitzgerald y G. Riordan (2016), "Using developmental evaluation as a design thinking tool for curriculum innovation in professional higher education", Higher Education Research and Development, 35(2), pp. 309-321, DOI: https://doi.org/10.1080/07 294360.2015.1087386.

Lohr, S. L. (2000), Muestreo. Diseño y análisis, México, Thomson Learning.

López, M. y K. Flores (2006), "Análisis de competencias a partir del uso de las TIC", Revista de Innovación Educativa, 0(5), pp. 36-55.

Malcolm, M. (2012), "Examining the implications of learner and supervisor perceptions of undergraduate dissertation research in Business and Management", Teaching in Higher Education, 17(5), pp. 565-576, DOI: https://doi.org/10.1080/13562517.2011.641005.

Manzano, N., A. Martín, M. Sánchez, A. Rísquez y M. Suárez (2012), "El rol del mentor en un proceso de mentoría universitaria", Educacion XXI, 15(2), pp. 93-118.

Mateo, J., A. Escofet, F. Martínez, J. Ventura y D. Vlachopoulos (2012), "The Final Year Project (FYP) in social sciences: establishment of its associated competences and evaluation standards", Studies in Educational Evaluation, 38(1), pp. 28-34.

Pathirage, C., R. Haigh, D. Amaratunga y D. Baldry (2007), "Enhancing the quality and consistency of undergraduate dissertation assessment", Quality Assurance in Education, 15(3), pp. 271-286, DOI: https:/ / doi.org/10.1108/09684880710773165.

Pathirage, C., S. Richard Haigh y D. Baldry (2004), "Improving dissertation assessment", <http://eprints. hud.ac.uk/id/eprint/22716/1/chp_04.pdf>.

Porter, B. (2011), "Millennial undergraduate research strategies in web and library information retrieval systems", Journal of Web Librarianship, 5(4), pp. 267-285.

Quevedo, T. y A. Rodrigues (2010), "El trabajo del mentor: análisis de los feedbacks de diarios reflexivos a lo largo de un proceso de mentoría en grupo", Revista Iberoamericana de Educación, 52(6), p. 4.

Ramírez, V. y G. Medina (2008), "Educación basada en competencias y el proyecto Tuning en Europa y Latinoamérica”, Ide@s CONCYTEG, 3(39), pp. 97-114.

Ravenscroft, S. P., J. E. Rebele, K. St. Pierre, K. y R. M. S. Wilson (2008), "The importance of accounting 
education research", fournal of Accounting Education, 26(4), pp. 180-187, DOI: https://doi.org/10.1016/j. jaccedu.2009.02.002.

Real Decreto 1393/2007, de 29 de octubre, por el que se establece la ordenación de las enseñanzas universitarias oficiales, publicado en: «BOE» núm. 260, de 30/10/2007. Ministerio de Educación y Ciencia. Referencia: BOE-A-2007-18770, < https://www.boe. es/eli/es/rd/2007/10/29/1393/con>.

Rebele, J. E. y E. K. St. Pierre (2015), "Stagnation in accounting education research", fournal of Accounting Education, 33(2), pp. 128-137, DOI: https://doi. org/10.1016/j.jaccedu.2015.04.003.

Rekalde, I. (2011), “CCómo afrontar el trabajo fin de grado? Un problema o una oportunidad para culminar con el desarrollo de las competencias", Revista Complutense de Educacion, 22(2), pp. 179-193, DOI: https://doi. org/10.5209/rev_RCED.2011.v22.n2.38488.

Romero, D. M., A. I. Corregidor-Sánchez y B. Polonio (2011), "Tutorización y evaluación del TFG-uso de rúbricas", en Margarita Marín Rodríguez y Ana Belén Morales Simancas (eds.), VII Intercampus 2011: "Trabajos de Fin de Grado y Máster: La evaluación global", ToledoEspaña, pp. 227-232, ISBN: 978-84-694-4404-7.

Rowe, C. y E. Okell (2009), "The "research-teaching nexus" and the learning-teaching relationship: who's in charge?", Arts and Humanities in Higher Education, vol. 8, núm. 2, pp. 180-190, DOI: https://doi. org/10.1177/1474022209102683.

Rowley, J. (2000), "Is higher education ready for knowledge management?", International fournal of Educational Management, 14(7), pp. 325-333, DOI: https://doi.org/10.1108/09513540010378978.

Rowley, J. y F. Slack (2004), "Conducting a literature review", Management Research News, 27(6), pp. 31-39, DOI: https://doi.org/10.1108/01409170410784185.

Ruíz-Lozano, M. y P. Tirado-Valencia (2016), "La percepción de los alumnos sobre la adquisición de competencias del Trabajo Fin de Grado en ADE. Un análisis en la Universidad Loyola Andalucía”, Educade, 7, pp. 19-39.
Rullan, M., M. Fernández, G. Estapé y M. D. Márquez (2010), "La evaluación de las competencias transversales en la materia Trabajos de Fin de Grado. Un estudio preliminar sobre la necesidad y oportunidad de establecer medios e instrumentos por ramas de conocimiento", Revista de Docencia Universitaria, 8(1), pp. 74-100.

Sanderson, P., D. Clewes y L. Hand (1998), "Action learning with business school undergraduates: three tutors use learning sets for project support", Assessment E Evaluation in Higher Education, 23(1), pp. 33-42, DOI: https://doi.org/10.1080/0260293980230103.

Saunders, M. N. K. y S. M. Davis (1998), "The use of assessment criteria to ensure consistency of marking: some implications for good practice", Quality Assurance in Education, 6(3), pp. 162-171, DOI: https://doi. org/10.1108/09684889810220465.

Starr-Glass, D. y T. Ali (2012), "Double standards: when an undergraduate dissertation becomes the object of two different assessment approaches", Assessment and Evaluation in Higher Education, 37(2), pp. 179-192, DOI: https://doi.org/10.1080/02602938.2010.515016.

Todd, M., P. Bannister y S. Clegg (2004), "Independent inquiry and the undergraduate dissertation: perceptions and experiences of final-year social science students", Assessment and Evaluation in Higher Education, 29(3), pp. 335-355.

Todd, M. J., K. Smith y P. Bannister (2006), "Supervising a social science undergraduate dissertation: staff experiences and perceptions", Teaching in Higher Education, 11(2), pp. 161-173, DOI: https://doi. org/10.1080/13562510500527693.

Torres, A. L., M. Badillo, N. O. Valentín y E. T. Ramírez (2014), "Las competencias docentes: el desafío de la educación superior", Innovación Educativa, 14(66), pp. 129-145.

Valderrama, E., M. Rullán, F. Sánchez, J. Pons, C. Mans, F. Giné y J. Bisbal (2010), "La evaluación de competencias en los trabajos fin de estudios", Revista Iberoamericana de Tecnologias del Aprendizaje, 5(3), pp. 107114, DOI: http://jenui2009.fib.upc.edu/. 
Vera, J., E. Briones y M. Sotos (2014), "Procesos de tutorización y evaluación del Trabajo de Fin de Grado (TFG): un estudio de dos años de seguimiento (20112013)", en XI Foro sobre la Evaluación de la calidad de la investigación y la educación superior, p. 440, DOI: http:// www.researchgate.net/publication/272504611.

Vera, J. y E. Briones (2016), "Un nuevo reto para las universidades: la evaluación de la calidad de la gestión de los Trabajos de Fin de Grado", Foro de Educación, 14(21), pp. 281-310, DOI: https://doi.org/http:// dx.doi.org/10.14516/fde.2016.014.021.014.

Vera, J. y E. Briones (2015), "Perspectiva del alumnado de los procesos de tutorización y evaluación de los trabajos de fin de grado", Cultura y Educación, 27(4), pp. 726-765, DOI: https://doi.org/10.1080/11356405.2015.1089391.

Vilardell i Riera, I. (2010), "Experiencia sobre el trabajo de fin de Grado en Administración y Dirección de Empresas", Educade: Revista de Educación En Contabilidad, Finanzas Y Administración de Empresas, 1, pp. 101-122.

Webster, F., D. Pepper y A. Jenkins (2000), "Assessing the undergraduate dissertation", Assessment and Evaluation in Higher Education, DOI: https://doi. org/10.1080/02602930050025042.

Woolhouse, M. (2002), "Supervising dissertation projects: expectations of supervisors and students", Innovations in Education and Teaching International, 39(2), pp. 137-144. 
Anexo 1. Centros de procedencia de las respuestas del cuestionario

\begin{tabular}{|c|c|c|c|c|c|c|c|c|}
\hline Universidad & $n$ & $\%$ & Universidad & $n$ & $\%$ & Universidad & $n$ & $\%$ \\
\hline A Coruña & 3 & 1.2 & Extremadura & 9 & 3.6 & Pablo Olavide & 14 & 5.7 \\
\hline Alcalá de Henares & 6 & 2.4 & Girona & 8 & 3.2 & País Vasco & 10 & 4.0 \\
\hline Alicante & 1 & 0.4 & Granada & 10 & 4.0 & Politécnica de Cartagena & 4 & 1.6 \\
\hline Almería & 2 & 0.8 & Huelva & 3 & 1.2 & Pontificia de Comillas & 1 & 0.4 \\
\hline Autónoma Barcelona & 2 & 0.8 & Illes Balears & 9 & 3.6 & Pública de Navarra & 1 & 0.4 \\
\hline Autónoma de Madrid & 1 & 0.4 & Jaén & 3 & 1.2 & Rey Juan Carlos & 2 & 0.8 \\
\hline Burgos & 5 & 2.0 & Jaume I & 2 & 0.8 & Rovira i Virgili & 1 & 0.4 \\
\hline Cádiz & 9 & 3.6 & La Laguna & 1 & 0.4 & Salamanca & 3 & 1.2 \\
\hline Cantabria & 3 & 1.2 & La Rioja & 3 & 1.2 & Santiago de Compostela & 6 & 2.4 \\
\hline Carlos III & 2 & 0.8 & Las Palmas de G.C. & 2 & 0.8 & Sevilla & 46 & 1.6 \\
\hline Castilla-La Mancha & 1 & 0.4 & León & 13 & 5.3 & UDIMA & 1 & 0.4 \\
\hline Católica de Valencia & 2 & 0.8 & Loyola Andalucía & 8 & 3.2 & UNED & 2 & 0.8 \\
\hline CEU San Pablo & 1 & 0.4 & Málaga & 4 & 1.6 & Valencia & 5 & 2.0 \\
\hline Complutense de Madrid & 7 & 2.8 & Miguel Hernández & 1 & 0.4 & Valladolid & 2 & 0.8 \\
\hline Deusto & 1 & 0.4 & Murcia & 7 & 2.8 & Vigo & 2 & 0.8 \\
\hline Europa de Valencia & 2 & 0.8 & Oviedo & 13 & 5.3 & Zaragoza & 3 & 1.2 \\
\hline Total & & & & & & & 247 & 100 \\
\hline
\end{tabular}

\section{Cómo citar este artículo:}

Donoso-Anes, José-Antonio, Francisco Serrano-Domínguez y José-Antonio Camúñez-Ruíz (2021), "Profesores universitarios de Contabilidad y el proceso de tutorización del trabajo fin de grado", Revista Iberoamericana de Educación Superior (RIES), vol. XII, núm. 34, pp. 46-71, DOI: https://doi.org/10.22201/iisue.20072872e.2021.34.978 [Consulta: fecha de última consulta]. 